\title{
Clinical Study \\ Skin Perfusion Pressure Is a Prognostic Factor in Hemodialysis Patients
}

\author{
Shingo Hatakeyama, ${ }^{1}$ Masaaki Saito, ${ }^{2}$ Kumiko Ishigaki, ${ }^{2}$ Hayato Yamamoto, ${ }^{3}$ \\ Akiko Okamoto, ${ }^{4}$ Yusuke Ishibashi, ${ }^{3}$ Hiromi Murasawa, ${ }^{3}$ Kengo Imanishi, ${ }^{3}$ Noriko Tokui, ${ }^{3}$ \\ Teppei Okamoto, ${ }^{3}$ Yuichiro Suzuki, ${ }^{3}$ Naoki Sugiyama, ${ }^{3}$ Atsushi Imai, ${ }^{3}$ Shigemasa Kudo, ${ }^{3}$ \\ Takahiro Yoneyama, ${ }^{3}$ Yasuhiro Hashimoto, ${ }^{4}$ Takuya Koie, ${ }^{3}$ Noritaka Kaminura, ${ }^{3}$ \\ Hisao Saitoh, ${ }^{4}$ Tomihisa Funyu, ${ }^{4}$ and Chikara Ohyama ${ }^{1,3}$
}

\author{
${ }^{1}$ Department of Advanced Transplant and Regenerative Medicine, Hirosaki University Graduate School of Medicine, \\ Hirosaki 036-8562, Japan \\ ${ }^{2}$ Department of Clinical Laboratory, Oyokyo Kidney Research Institute, Hirosaki 036-8243, Japan \\ ${ }^{3}$ Department of Urology, Hirosaki University Graduate School of Medicine, Hirosaki 036-8562, Japan \\ ${ }^{4}$ Department of Urology, Oyokyo Kidney Research Institute, Hirosaki 036-8243, Japan
}

Correspondence should be addressed to Chikara Ohyama, coyama@cc.hirosaki-u.ac.jp

Received 3 December 2011; Accepted 30 January 2012

Academic Editor: David B. Kershaw

Copyright (C) 2012 Shingo Hatakeyama et al. This is an open access article distributed under the Creative Commons Attribution License, which permits unrestricted use, distribution, and reproduction in any medium, provided the original work is properly cited.

Peripheral arterial disease (PAD) is common in hemodialysis patients and predicts a poor prognosis. We conducted a prospective cohort study to identify risk factors for PAD including skin perfusion pressure (SPP) in hemodialysis patients. The cohort included 373 hemodialysis patients among 548 patients who received hemodialysis at Oyokyo Kidney Research Institute, Hirosaki, Japan from August 2008 to December 2010. The endpoints were lower limb survival (peripheral angioplasty or amputation events) and overall survival of 2 years. Our results showed that $<70 \mathrm{mmHg}$ SPP was a poor prognosis for the lower limb survival and overall survival. We also identified age, history of cardiovascular disease, presence of diabetes mellitus, smoking history, and SPP $<70 \mathrm{mmHg}$ as independent risk factors for lower limb survival and overall survival. Then, we constructed risk criteria using the significantly independent risk factors. We can clearly stratify lower limb survival and overall survival of the hemodialysis patients into 3 groups. Although the observation period is short, we conclude that SPP value has the potential to be a risk factor that predicts both lower limb survival and the prognosis of hemodialysis patients.

\section{Introduction}

Peripheral arterial disease (PAD) is common in hemodialysis patients and predicts a poor prognosis [1]. Data from the Dialysis Outcomes and Practice Patterns Study have shown that PAD is associated with an increased risk for allcause mortality and cardiovascular disease (CVD) [2]. Early detection of PAD is important to improve the prognosis in hemodialysis patients.

Various noninvasive methods of ankle brachial pressure index (ABI), toe blood pressure index (TBI), and transcutaneous oxygen pressure $\left(\mathrm{tcPO} \mathrm{PO}_{2}\right)$ have been widely used to diagnose PAD. An ABI is currently used worldwide for evaluating PAD; a $<0.9$ value has $>98 \%$ specificity for detecting PAD. However, high ABI values of $>1.3$ are common in patients with diabetes mellitus (DM) or renal failure, because they may have calcification in lower leg artery, causing a falsely raised ABI [3]. False negative ABI results occur in $17 \%-24 \%$ of limbs of DM and hemodialysis patients $[4,5]$. In contrast, measuring skin perfusion pressure (SPP) using laser Doppler is a noninvasive method that measures microcirculatory pressure of the artery at the skin level. It can detect the movement of red blood cells by slowly decreasing the inflation-cuff pressure at the site of measurement. SPP has proven to be beneficial for assessing the ischemic severity of lower limb $[6,7]$, selecting of suitable 
degree of amputation $[8,9]$, and useful for judgment of likelihood that ischemic foot ulcers will recover $[10,11]$. SPP measurements have advantages for predicting wound healing at the amputation edge in chronic critical limb ischemia with a cutoff value of $<30 \mathrm{~mm} \mathrm{Hg}$ [12]. Okamoto et al. reported the superiority of SPP measurements for detecting PAD in hemodialysis patients with a cutoff value of $50 \mathrm{~mm} \mathrm{Hg}$ [13]. Because SPP indicates the final pathway of capillary flow through the skin with a laser Doppler probe, it has potential to determine severe limb ischemia status with calcification in hemodialysis patients.

Chronic kidney disease (CKD) and PAD independently predict mortality. Both CKD and PAD patients have a significantly higher risk for death (odds ratio, 2.4) [14] and longterm survival is dismal for major lower extremity amputation patients with end-stage renal disease [15]. However, not much is known about SPP values for prognosis, and no attempts have been made to classify the multiple risks of PAD patients undergoing hemodialysis. In this study, we hypothesized that SPP value has potential to predict patient's prognosis, and we determined the SPP cutoff value for the lower limb survival and overall survival and the PAD risk classification for hemodialysis patients.

\section{Materials and Methods}

2.1. Subjects. From August 2008 to December 2010, 548 patients underwent hemodialysis at the Oyokyo Kidney Research Institute, Hirosaki, Japan. Among these, 373 hemodialysis patients who agreed to enter the present study were enrolled as the cohort. We excluded these patients who were not able to measure because of restless leg syndrome or out of follow-up, or refused to measure SPP. We measured SPP in August 2008 and prospectively observed lower limb survival (peripheral angioplasty or amputation) and overall survival for 2 years. This study was approved by the institutional ethical committee of Oyokyo Kidney Research Institute. Informed consent was obtained from all patients.

2.2. SPP Measurements. SPP measurements of the soles of the feet were conducted $1 \mathrm{~h}$ after the hemodialysis session. SPP was measured with a laser Doppler probe enclosed within the bladder and a cuff wrapped around the patient's foot sole (foot arch) using SensiLase PAD3000 (Kaneka Medix Corp., Osaka, Japan). The patients were positioned in a supine position at room temperature [16]. To evaluate ischemic status of both legs, an average of 2 feet was regarded as the SPP value for each patient.

2.3. Patient Classification. We measured SPP in 35 healthy subjects (mean age: $50.8 \pm 7.9$ years, range: $38-76$ years, number smoking: 20) to determine a SPP reference range. The healthy subjects were free of DM, hypertension, hyperlipidemia, or other diseases. We considered an SPP value of 50 and 70 as cutoff values, because SPP from the 35 healthy subjects showed an average value of $75.3 \pm 9.2 \mathrm{~mm} \mathrm{Hg}$, and Okamoto et al. [13] reported $74.8 \pm 28.5$ as a healthy SPP value ( $n=26$; age, $59 \pm 10.7$ years). Based on a healthy
SPP value, we categorized the patients into three SPP groups: Group 1, <50; Group 2, $50 \leq$ SPP $<70$; and Group 3, $\mathrm{SPP} \geq 70$. A foot $\mathrm{SPP}<50 \mathrm{~mm} \mathrm{Hg}$ was candidate for PAD treatment. We started anticoagulant or antiplatelet treatment and performed PAD examination (digital subtraction angiography). If there were stenotic lesions, percutaneous transluminal angioplasty was performed.

2.4. Evaluation. The background clinical data, gender, concomitant drugs, smoking habit, and incidences of peripheral angioplasty or amputation were compared among the groups using the chi-square test. Age and other biochemical parameters were expressed as mean $\pm \mathrm{SD}$, and statistical differences were determined by the overall ANOVA, Student's $t$-test, or chi-square analysis. Cumulative lower limb survival and overall survival rates were plotted using the KaplanMeier method, and the intergroup differences were tested with the log-rank test. $P<0.05$ was considered significant. A Cox regression model, adjusted for these factors, was also performed. The data used in the analyses included age, gender, dialysis duration, concomitant drugs (activated vitamin $\mathrm{D}$, anticoagulants, or antiplatelet drugs), presence of CVD (heart failure, myocardial infarction, and angina pectoris), DM, SPP value, and smoking. Based on the independent risk factors identified by the Cox regression analysis, we determined the risk classification for lower limb survival and overall survival by the numbers of risk factors. All analyses were performed using SPSS ver. 12.0 (SPSS Inc., Chicago, IL, USA).

\section{Results}

Table 1 shows the characteristics of the 373 patients. Significant differences were observed among the groups for the presence of CVD, DM, use of activated vitamin D, use of anticoagulant or antiplatelet drugs, peripheral angioplasty or amputation events, and cause of death, but there were no differences in cause of deaths. Figure 1 shows lower limb survival and overall survival in the three groups. Group 1 $($ SPP $<50)$ showed poor lower limb survival compared to that in the other groups. Group $2(50 \leq \mathrm{SPP}<70)$ showed better survival compared to that in Group $1(P<0.0001)$, but a poor prognosis for lower limb survival compared to that in Group 3 (SPP $\geq 70)(P=0.0005)$. No significant difference in overall survival was observed between Groups 1 and $2(P=0.2519)$. From these results, we recategorized the patients characteristics with an SPP $<70$ (Groups 1 and 2) and $>70$ (Group 3) (Table 2). The numbers of patients with CVD, DM, use of activated vitamin D, use of anticoagulant or antiplatelet drugs, peripheral angioplasty or amputation events, or death were significantly higher in Groups 1 and 2 than those in Group 3. Lower limb survival and overall survival were significantly poorer in Groups 1 and 2 compared with those in Group 3 (Figure 2). The Cox regression analysis revealed that an $\mathrm{SPP}<70$ and the presence of CVD, DM, and smoking were independent factors for lower limb survival. Age $\geq 71.4$ years, an SPP $<70$, and the presence of DM and smoking were independent factors for overall survival (Table 3). We used average of SPP vale 
TABLE 1: Characteristics of the 373 hemodialysis patients. We categorized the patients into three skin perfusion pressure (SPP) groups: Group $1,<50$; Group 2, $50 \leq \mathrm{SPP}<70$; and Group 3, SPP $\geq 70$, based on a healthy SPP value from volunteers. $P$ value refers to overall ANOVA or chi-square analysis.

\begin{tabular}{|c|c|c|c|c|c|}
\hline & ALL & $\begin{array}{l}\text { Group } 1 \\
\mathrm{SPP}<50\end{array}$ & $\begin{array}{c}\text { Group } 2 \\
50 \leq \mathrm{SPP}<70\end{array}$ & $\begin{array}{l}\text { Group } 3 \\
\mathrm{SPP} \geq 70\end{array}$ & $\begin{array}{c}P \text { value } \\
\text { (ANOVA) }\end{array}$ \\
\hline$n$ & 373 & $30(8 \%)$ & $84(23 \%)$ & $259(69 \%)$ & \\
\hline Age & $71.4 \pm 9.7$ & $71.5 \pm 11.4$ & $72.6 \pm 11.1$ & $71.0 \pm 9.0$ & 0.437 \\
\hline Gender (M/F) & $207 / 166$ & $15 / 15$ & $50 / 34$ & $142 / 117$ & 0.617 \\
\hline Dialysis duration (Month) & $100.4 \pm 77.1$ & $103.6 \pm 88.1$ & $100.6 \pm 81.5$ & $100.0 \pm 74.6$ & 0.970 \\
\hline $\operatorname{CVD}(+)$ & $164(44 \%)$ & $13(43 \%)$ & $48(57 \%)$ & $103(40 \%)$ & 0.020 \\
\hline $\mathrm{DM}(+)$ & $171(46 \%)$ & $21(70 \%)$ & $48(57 \%)$ & $102(39 \%)$ & $<0.001$ \\
\hline Use of activated vitamin $\mathrm{D}(+)$ & $267(72 \%)$ & $23(77 \%)$ & $56(67 \%)$ & $217(73 \%)$ & 0.003 \\
\hline Current smoking $(+)$ & $55(15 \%)$ & $6(21 \%)$ & $16(20 \%)$ & $33(13 \%)$ & $<0.001$ \\
\hline Baseline SPP ( $\mathrm{mm} \mathrm{Hg}$ ) & $77.9 \pm 21.1$ & $35.6 \pm 8.2$ & $61.0 \pm 5.7$ & $88.3 \pm 14.6$ & $<0.001$ \\
\hline Use of anticoagulant, antiplatelet & $173(46 \%)$ & $20(67 \%)$ & $66(79 \%)$ & $87(34 \%)$ & $<0.001$ \\
\hline Periferal angioplasty & $11(2.9 \%)$ & $6(20 \%)$ & $4(4.8 \%)$ & $1(0.4 \%)$ & $<0.001$ \\
\hline Amputation & $6(1.6 \%)$ & $5(17 \%)$ & $1(1.2 \%)$ & $0(0 \%)$ & $<0.001$ \\
\hline Death & $53(14 \%)$ & $9(30 \%)$ & $16(19 \%)$ & $28(11 \%)$ & 0.006 \\
\hline CVD & 23 & 4 & 8 & 11 & 0.055 \\
\hline Infection & 10 & 0 & 3 & 7 & 0.582 \\
\hline Cancer & 10 & 1 & 3 & 6 & 0.804 \\
\hline Others & 10 & 4 & 2 & 4 & $<0.001$ \\
\hline
\end{tabular}

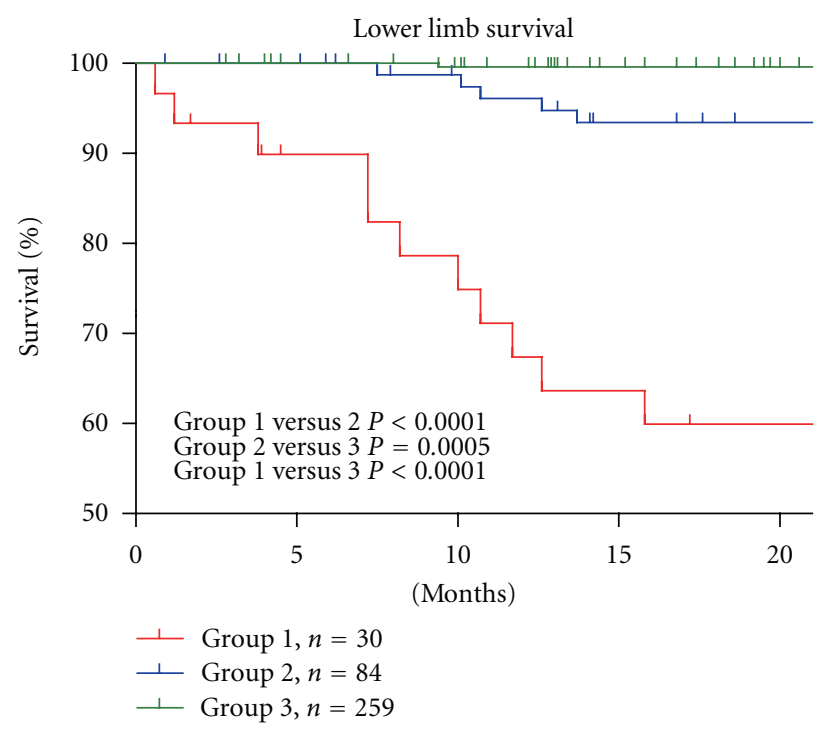

(a)

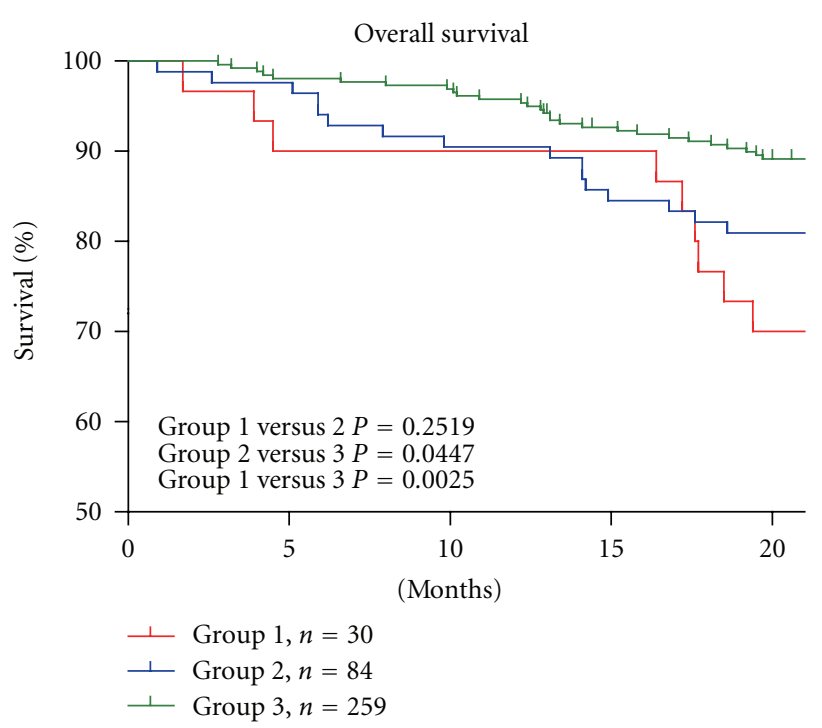

(b)

FIGURE 1: Lower limb survival and overall survival in the three groups. Patients were categorized according to the skin perfusion pressure (SPP) value: Group 1, <50; Group 2, $50 \leq \mathrm{SPP}<70$; and Group 3, SPP $\geq 70$. Group 1 showed poorer lower limb survival compared to other groups. Group 2 showed better survival compared to Group $1(P<0.0001)$ but a poor prognosis for lower limb survival compared to Group $3(P=0.0005)$. No significant difference in overall survival was observed between Groups 1 and $2(P=0.2519)$.

from both feet because the result from worst feet for lower limb survival and overall survival showed similar outcomes (Figure 3).

Based on the independent risk factors, we categorized the three groups according to the number of risk factors. Patients with zero or one risk factor, two risk factors, and three or four risk factors were regarded as low-risk, intermediaterisk, and high-risk groups, respectively. Cumulative lower limb survival and overall survival rates were plotted using the Kaplan-Meier method according to the risk classification (Figure 4). Lower limb survival and overall survival at 1 and 2 years were significantly lower in the high-risk group than 
TABLE 2: Recategorization of patient characteristics with a skin perfusion pressure (SPP) cutoff value of $70 \mathrm{~mm} \mathrm{Hg}$. We recategorized patients into two SPP groups: Groups 1 and 2: $<70$ and Group 3: $\geq 70$. $P$ value refers to Student's $t$-test or chi-square analysis.

\begin{tabular}{|c|c|c|c|}
\hline & $\begin{array}{c}\text { Groups } 1 \text { and } 2 \\
\text { SPP }<70\end{array}$ & $\begin{array}{l}\text { Group } 3 \\
\mathrm{SPP} \geq 70\end{array}$ & $\begin{array}{c}P \text { value } \\
\text { Group } 1 \text { and } 2 \text { versus } 3\end{array}$ \\
\hline$n$ & $114(31 \%)$ & $259(69 \%)$ & \\
\hline Age & $72.3 \pm 11.1$ & $71.0 \pm 9.0$ & 0.282 \\
\hline Gender (M/F) & $65 / 49$ & $142 / 117$ & 0.695 \\
\hline Dialysis duration (Month) & $101.4 \pm 82.9$ & $100.0 \pm 74.6$ & 0.875 \\
\hline $\operatorname{CVD}(+)$ & $61(54 \%)$ & $103(40 \%)$ & 0.014 \\
\hline $\mathrm{DM}(+)$ & $69(61 \%)$ & $102(39 \%)$ & $<0.001$ \\
\hline Use of activated vitamin $\mathrm{D}(+)$ & $79(69 \%)$ & $217(73 \%)$ & 0.001 \\
\hline Current smoking $(+)$ & $22(20 \%)$ & $33(13 \%)$ & 0.100 \\
\hline Baseline SPP (mm Hg) & $54.3 \pm 12.9$ & $88.3 \pm 14.6$ & $<0.001$ \\
\hline Use of anticoagulant, antiplatelet & $86(75 \%)$ & $87(34 \%)$ & $<0.001$ \\
\hline Intervention or amputation & $16(14 \%)$ & $1(0.4 \%)$ & $<0.001$ \\
\hline Death & $25(22 \%)$ & $28(11 \%)$ & 0.005 \\
\hline CVD & 12 & 11 & 0.523 \\
\hline Infection & 3 & 7 & 0.227 \\
\hline Cancer & 4 & 6 & 0.614 \\
\hline Others & 6 & 4 & 0.367 \\
\hline
\end{tabular}

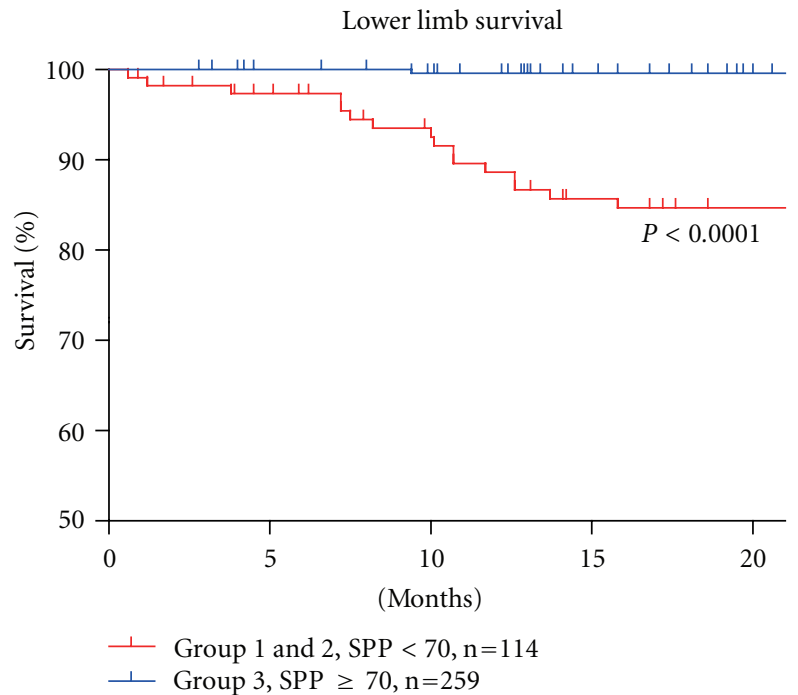

(a)

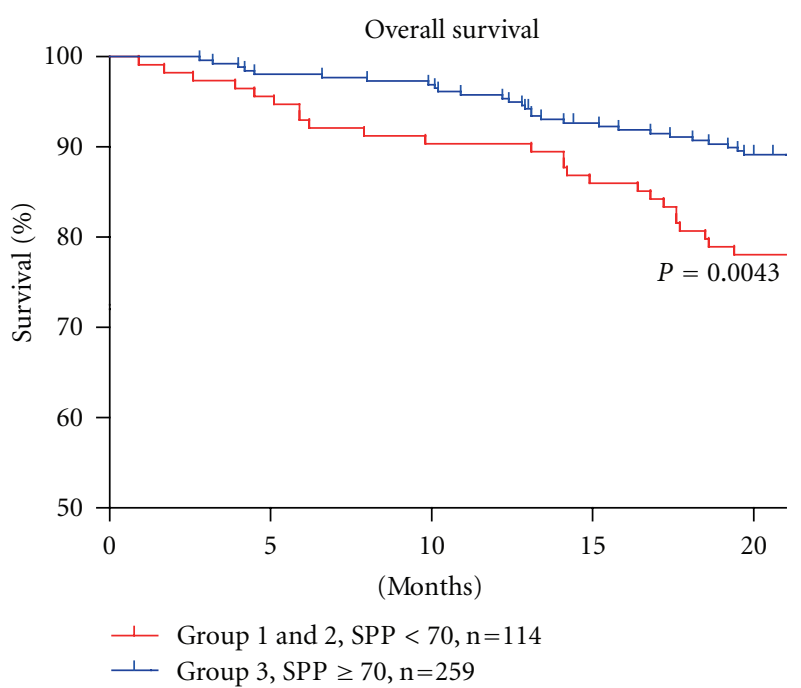

(b)

FIGURE 2: Lower limb survival and overall survival in Groups 1 and 2 versus 3. Patients were recategorized according to their SPP values: Groups 1 and 2: $<70$ and Group 3: SPP $\geq 70$. Lower limb survival and overall survival were significantly poorer in Groups 1 and $2(P<$ $0.0001)$ compared with those in Group $3(P=0.0043)$.

those in the other groups (Table 4). Lower limb survival and overall survival rates at 1 year were $83.0 \%$ and $85.7 \%$ and at 2 years were $76 \%$ and $61.2 \%$ in the high-risk group patients, respectively.

\section{Discussion}

Both CKD and PAD patients have a significantly higher risk for death [14], and an early detection of PAD in hemodialysis patients is extremely important. Because calcification in lower leg arteries causes a false negative ABI, SPP is a more sensitive and specific method for detecting PAD in hemodialysis patients, and an SPP of $50 \mathrm{~mm} \mathrm{Hg}$ has been suggested as the PAD cutoff value in these patients [13]. Our result showed that Group $1(\mathrm{SPP}<50)$ had high morbidity and mortality with the $20 \%$ of amputation and $67 \%$ of peripheral angioplasty. However, starting treatment from an SPP of $50 \mathrm{~mm} \mathrm{Hg}$ may be late in hemodialysis patients from the viewpoint of early detection and interposition to improve prognosis. We observed a better outcome for lower limb 


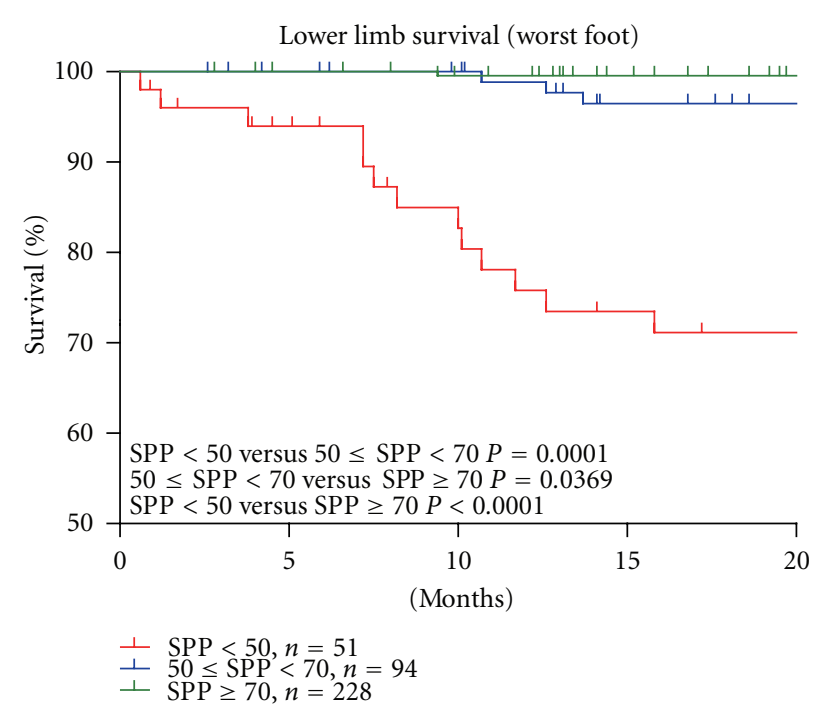

(a)

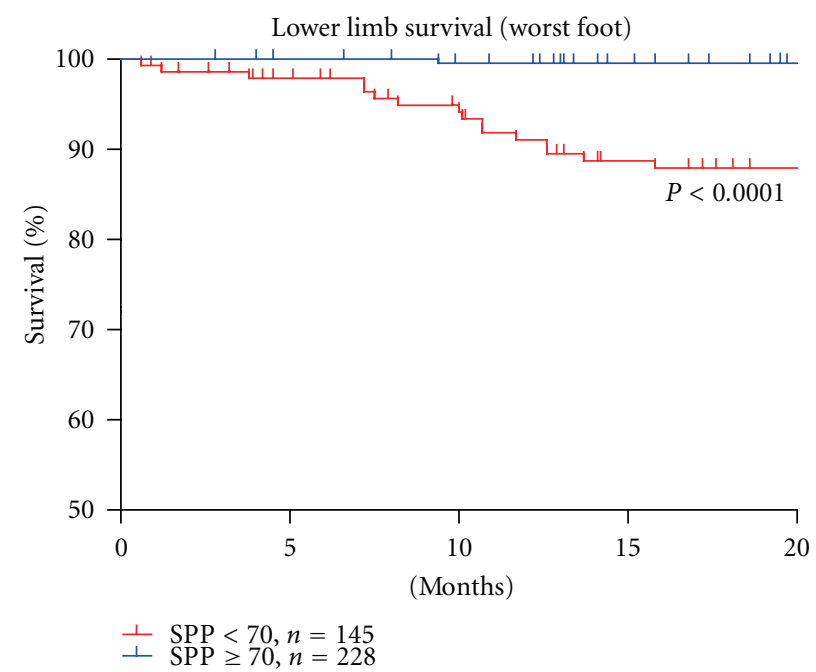

(c)

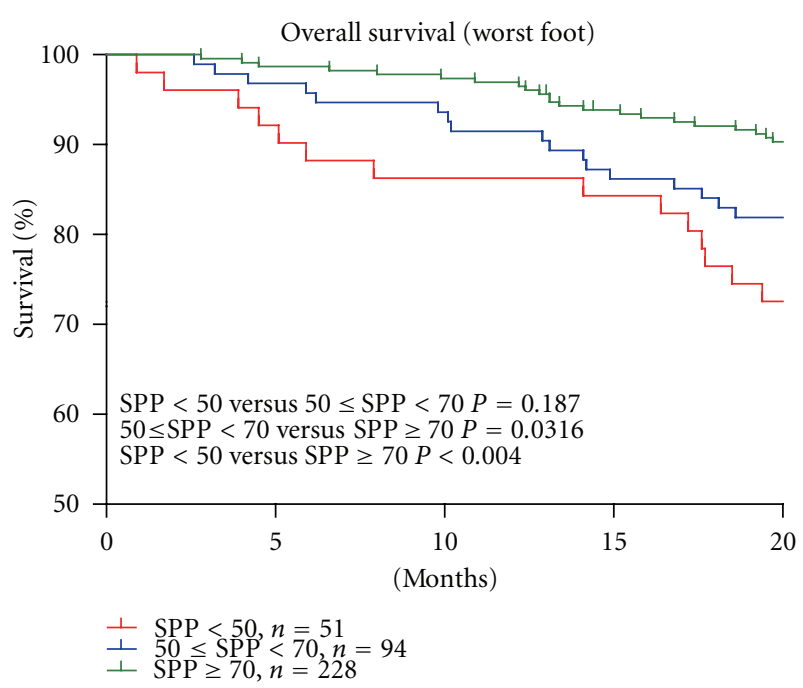

(b)

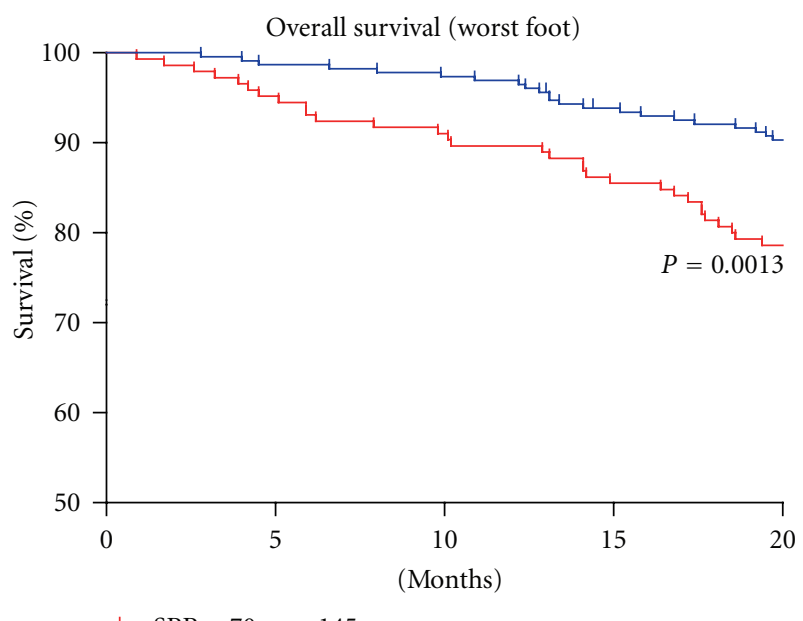

$\perp \mathrm{SPP}<70, n=145$
$\perp \mathrm{SPP} \geq 70, n=228$ survival in Group $2(50 \leq \mathrm{SPP}<70)$ compared with that in Group 1, but overall survival was not significantly different between Groups 1 and $2(\mathrm{SPP}<70)(P=0.2519$, Figure 1$)$. Because SPP reflects the final capillary flow though the skin, an SPP $<70$ may indicate systematic circulatory failure in hemodialysis patients. When we compared Groups 1 and 2 with 3 (SPP $\geq 70$ ), many critical parameters (presence of CVD, DM, use of activated vitamin D, use of anticoagulant or antiplatelet drugs, peripheral angioplasty or amputation events, and death) were significantly higher in Groups 1 and 2 than in 3 . This suggests that patients with an SPP $<70$ have many risk factors for high mortality.

To address the worst foot influences, we analyzed lower limb survival and overall survival, taking the SPP value from worst foot sole (Figure 3). The differences of SPP value between two feet were $11.1 \pm 9.5 \mathrm{mmHg}$ (median 9.0, maximum $56 \mathrm{~mm} \mathrm{Hg}$ ). The Kaplan-Meier curves showed similar outcomes between worst foot and average of both feet in survivals. It means that an average of SPP form both feet is adequate marker to express general ischemic status of hemodialysis patients, and an average of $\mathrm{SPP}<70$ is the risk factor for lower limb survival and overall survival.

In an attempt to classify the multiple risks for prognosis in hemodialysis patients using a Cox regression analysis, we identified SPP $<70$ as one of the independent risk factors for lower limb survival and prognosis, similar to well-known risk factors for CVD or DM. A 2-year overall survival of $61.2 \%$ in high-risk patients was remarkably low. These results suggest 


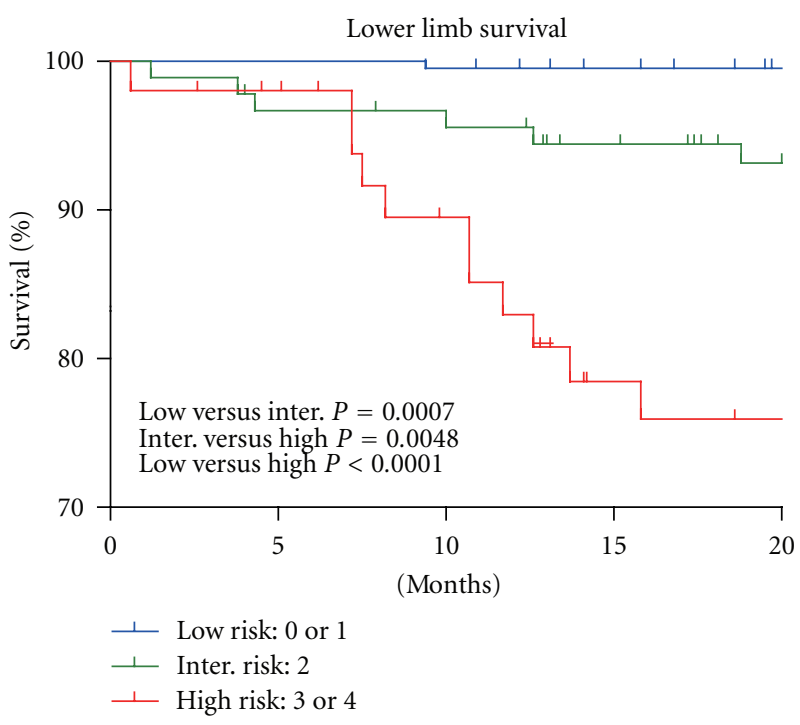

(a)

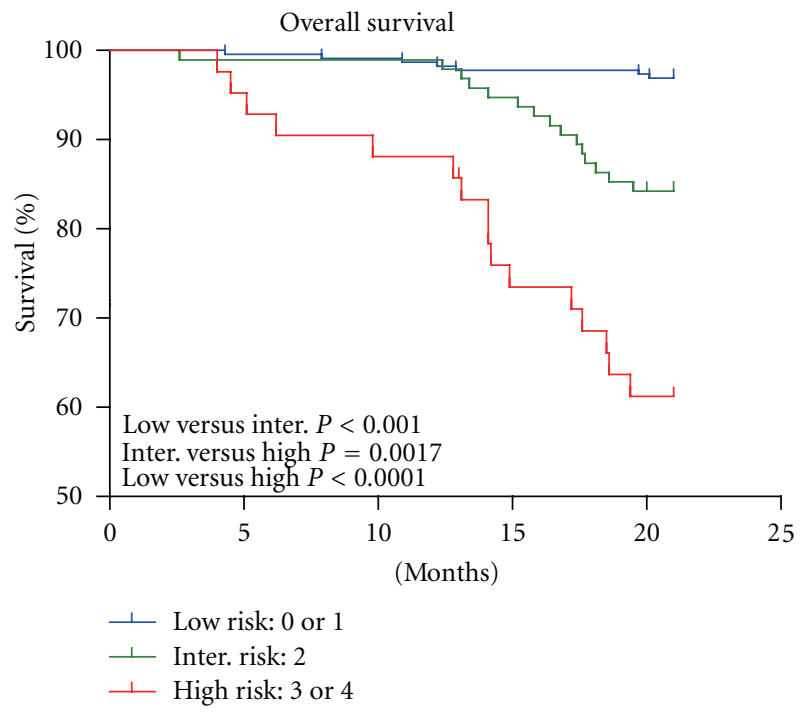

(b)

FIGURE 4: Lower limb survival and overall survival based on the risk classification. We categorized the three groups according to the number of risk factors based on a Cox regression analysis. Patients with zero or one risk factor, two risk factors, and three or four risk factors were regarded as the low-risk, intermediate-risk, and high-risk groups, respectively. Lower limb survival and overall survival were significantly lower in the high-risk group than in the other groups.

TABLE 3: Independent risk factors for lower limb survival and overall survival by Cox regression analysis. SPP $<70$, presence of CVD, DM, and smoking showed significantly increase the risk for lower limb survival (hazard ratios increased 4.722, 3.407, 4.050, 3.225 times, resp.), and age $\geq 71.4$, SPP $<70$, presence of $\mathrm{DM}$, and smoking showed significantly increase the risk for overall survival (hazard ratios increased 1.121, 1.209, 1.028, 4.521 times, resp.). $\left({ }^{*} \mathrm{HR}\right.$ : hazard ratio; ${ }^{* *} \mathrm{CI}$ : confidence interval).

\begin{tabular}{lcccc}
\hline Lower limb survival & $P$ value & HR $^{*}$ & \multicolumn{2}{c}{$95 \%$ CI $^{* *}$} \\
\hline $\begin{array}{l}\text { SPP, } \geq 70 \text { versus }<70 \\
\text { CVD, without versus }\end{array}$ & 0.007 & 4.722 & 1.539 & 14.490 \\
$\begin{array}{l}\text { with } \\
\text { DM, without versus } \\
\text { with }\end{array}$ & 0.027 & 3.407 & 1.146 & 10.124 \\
$\begin{array}{l}\text { Smoking, without } \\
\text { versus with }\end{array}$ & 0.035 & 4.050 & 1.102 & 14.876 \\
\hline $\begin{array}{l}\text { Overall survival } \\
\text { Age, }<71.4 \text { versus }\end{array}$ & P value & HR & \multicolumn{2}{c}{$95 \%$ CI } \\
$\begin{array}{l}\geq 71.4 \\
\text { SPP, } \geq 70 \text { versus }<70\end{array}$ & 0.001 & 1.073 & 1.121 & 1.028 \\
$\begin{array}{l}\text { DM, without versus } \\
\text { with }\end{array}$ & 0.042 & 2.239 & 1.028 & 4.875 \\
$\begin{array}{l}\text { Smoking, without } \\
\text { versus with }\end{array}$ & 0.000 & 9.135 & 4.521 & 18.460 \\
\hline
\end{tabular}

that early detection of peripheral hypoperfusion and higher cut-off ( SPP $<70$, instead of $<50$ ) is critical to improve survival in hemodialysis patients in clinical practices.

Our study had several limitations. Because of the observational nature of this study at a single institute, our observations cannot be generalized to the broader question
TABLe 4: Two-year outcomes of lower limb survival and overall survival by risk classification. Lower limb and overall survival were significantly lower in the high-risk group compared to those in the other groups.

\begin{tabular}{lcc}
\hline Lower limb survival & 1 year & 2 years \\
\hline Low risk & $99.5 \%$ & $99.5 \%$ \\
Intermediate risk & $95.6 \%$ & $93.2 \%$ \\
High risk & $83 \%$ & $76 \%$ \\
\hline Overall survival & 1 year & 2 years \\
\hline Low risk & $98.2 \%$ & $96.9 \%$ \\
Intermediate risk & $97.8 \%$ & $84.2 \%$ \\
High risk & $85.7 \%$ & $61.2 \%$ \\
\hline
\end{tabular}

of prognostic potential of SPP in PAD patients who are undergoing hemodialysis. We have no clear answer that why SPP was not a significant predictor of CVD-specific death while SPP was a significant predictor of overall death. It may because of short time follow-up or residual confounding and missing data may have introduced bias. However, this is the first study investigating an association between SPP and mortality with a risk classification. Therefore, our followup study is needed to confirm the relationship among SPP, prognosis, and efficacy of the risk classification in hemodialysis patients including other confounding: a measure of hypertension, number of hypertensive medications, a measure of cholesterol and diabetes management, and calcium, phosphate, and intact PTH levels.

In conclusion, we showed that an SPP $<70$ was a risk factor for lower limb survival and overall survival in hemodialysis patients. To our knowledge, this is the first report to determine the SPP value for a prognosis and risk 
classification. The SPP value, age, presence of CVD, DM, and smoking were associated with a poor prognosis. Early detection of peripheral hypoperfusion and interposition is critical to improve survival in hemodialysis patients.

\section{Conflict of Interests}

All the authors declared that they have no conflict of interests.

\section{Acknowledgments}

The authors thank Mrs. Saori Araya for her invaluable help with the original data. This paper was supported by a Grantsin-Aid for Scientific Research 23791737 from the Japan Society for the Promotion of Science.

\section{References}

[1] B. G. Jaar, B. C. Astor, J. S. Berns, and N. R. Powe, "Predictors of amputation and survival following lower extremity revascularization in hemodialysis patients," Kidney International, vol. 65, no. 2, pp. 613-620, 2004.

[2] S. Rajagopalan, S. Dellegrottaglie, A. L. Furniss et al., "Peripheral arterial disease in patients with end-stage renal disease: observations from the Dialysis Outcomes and Practice Patterns Study (DOPPS)," Circulation, vol. 114, no. 18, pp. 19141922, 2006.

[3] A. T. Hirsch, Z. J. Haskal, N. R. Hertzer et al., "ACC/AHA 2005 Practice Guidelines for the management of patients with peripheral arterial disease (lower extremity, renal, mesenteric, and abdominal aortic): a collaborative report from the American Association for Vascular Surgery/Society for Vascular Surgery, Society for Cardiovascular Angiography and Interventions, Society for Vascular Medicine and Biology, Society of Interventional Radiology," Circulation, vol. 113, no. 11, pp. e463-654, 2006.

[4] S. A. Carter, "Ankle and toe systolic pressures comparison of value and limitations in arterial occlusive disease," International Angiology, vol. 11, no. 4, pp. 289-297, 1992.

[5] J. Larsson, J. Apelqvist, J. Castenfors, C. D. Agardh, and A. Stenstrom, "Distal blood pressure as a predictor for the level of amputation in diabetic patients with foot ulcer," Foot and Ankle, vol. 14, no. 5, pp. 247-253, 1993.

[6] P. Holstein, J. Trap Jensen, H. Bagger, and B. Larsen, "Skin perfusion pressure measured by isotope washout in legs with arterial occlusive disease. Evaluation of different tracers, comparison with segmental systolic pressure, angiography and transcutaneous oxygen tension and variations during changes in systemic blood pressure," Clinical Physiology, vol. 3, no. 4, pp. 313-324, 1983.

[7] J. J. Castronuovo Jr., T. S. Pabst, D. P. Flanigan, and L. G. Foster, "Noninvasive determination of skin perfusion pressure using a laser Doppler," Journal of Cardiovascular Surgery, vol. 28, no. 3, pp. 253-257, 1987.

[8] P. Holstein, P. Sager, and N. A. Lassen, "Wound healing in below-knee amputations in relation to skin perfusion pressure," Acta Orthopaedica Scandinavica, vol. 50, no. 1, pp. 4958, 1979.

[9] H. R. I. Jorgensen, N. Wisbech Pedersen, H. Oxhoj, and V. Damholt, "Selection of amputation level in ischemia. Skin blood flow and perfusion pressure equally predictive," Acta Orthopaedica Scandinavica, vol. 61, no. 1, pp. 62-65, 1990.

[10] I. Faris and H. Duncan, "Skin perfusion pressure in the prediction of healing in diabetic patients with ulcers or gangrene of the foot," Journal of Vascular Surgery, vol. 2, no. 4, pp. 536540, 1985.

[11] B. Guillot, M. Dandurand, and J. J. Guilhou, "Skin perfusion pressure in leg ulcers assessed by photoplethysmography," International Angiology, vol. 7, no. 2, supplement, pp. 33-34, 1988.

[12] J. J. Castronuovo Jr., H. M. Adera, J. M. Smiell, and R. M. Price, "Skin perfusion pressure measurement is valuable in the diagnosis of critical limb ischemia," Journal of Vascular Surgery, vol. 26, no. 4, pp. 629-637, 1997.

[13] K. Okamoto, M. Oka, K. Maesato et al., "Peripheral Arterial Occlusive Disease Is More Prevalent in Patients With Hemodialysis: Comparison With the Findings of Multidetector-Row Computed Tomography," American Journal of Kidney Diseases, vol. 48, no. 2, pp. 269-276, 2006.

[14] Y. P. Liew, J. R. Bartholomew, S. Demirjian, J. Michaels, and M. J. Schreiber, "Combined effect of chronic kidney disease and peripheral arterial disease on all-cause mortality in a highrisk population," Clinical Journal of the American Society of Nephrology, vol. 3, no. 4, pp. 1084-1089, 2008.

[15] B. Aulivola, C. N. Hile, A. D. Hamdan et al., "Major lower extremity amputation: outcome of a modern series," Archives of Surgery, vol. 139, no. 4, pp. 395-399, 2004.

[16] T. Yamada, T. Ohta, H. Ishibashi et al., "Clinical reliability and utility of skin perfusion pressure measurement in ischemic limbs-Comparison with other noninvasive diagnostic methods," Journal of Vascular Surgery, vol. 47, no. 2, pp. 318-323, 2008. 


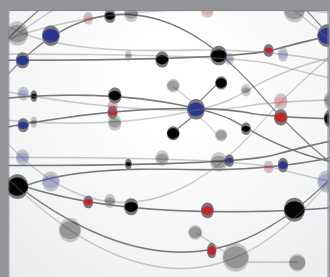

The Scientific World Journal
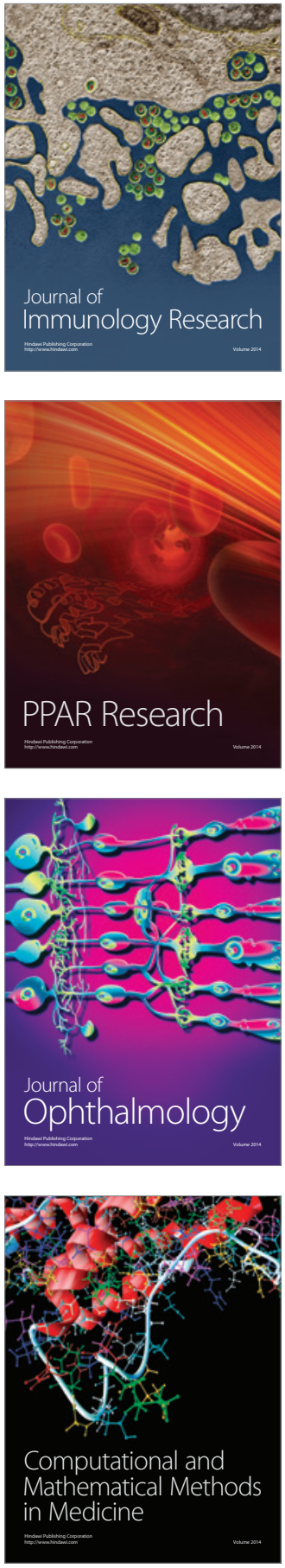

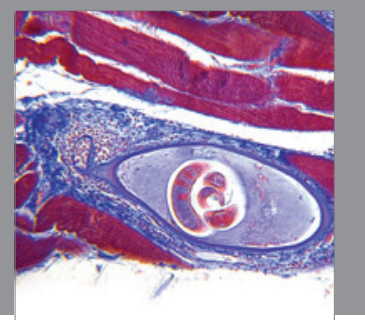

Gastroenterology

Research and Practice
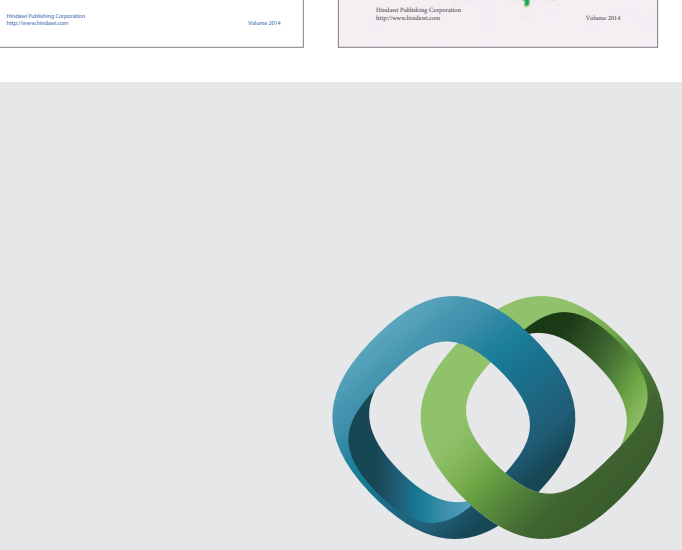

\section{Hindawi}

Submit your manuscripts at

http://www.hindawi.com
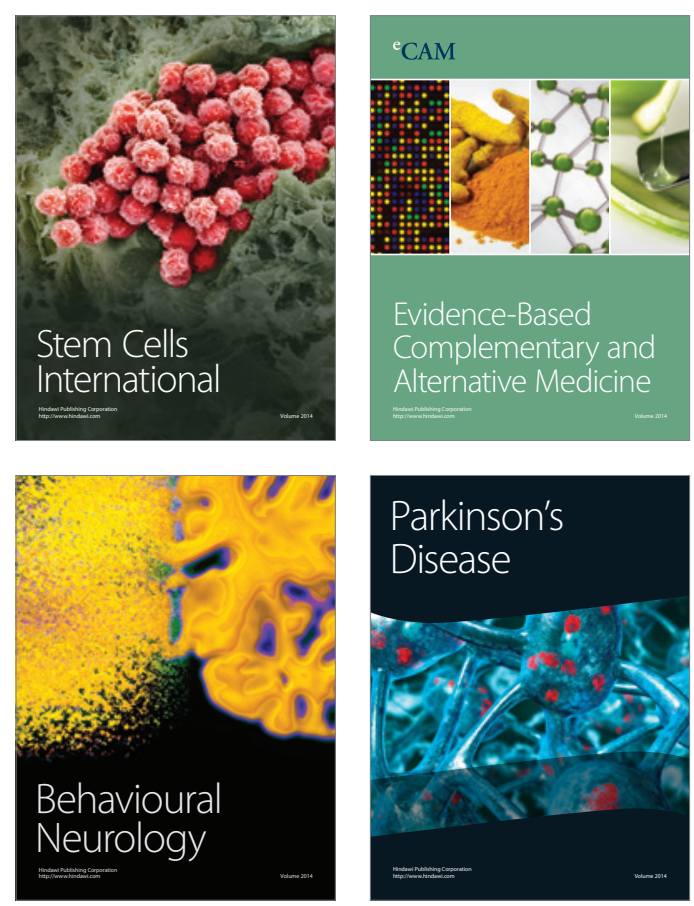

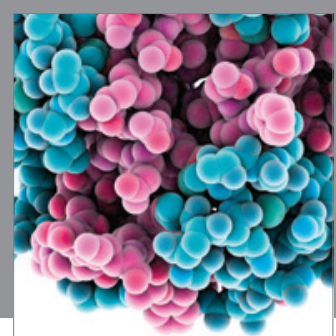

Journal of
Diabetes Research

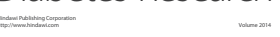

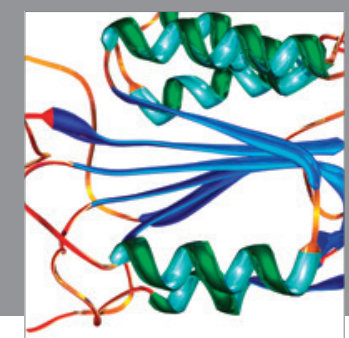

Disease Markers
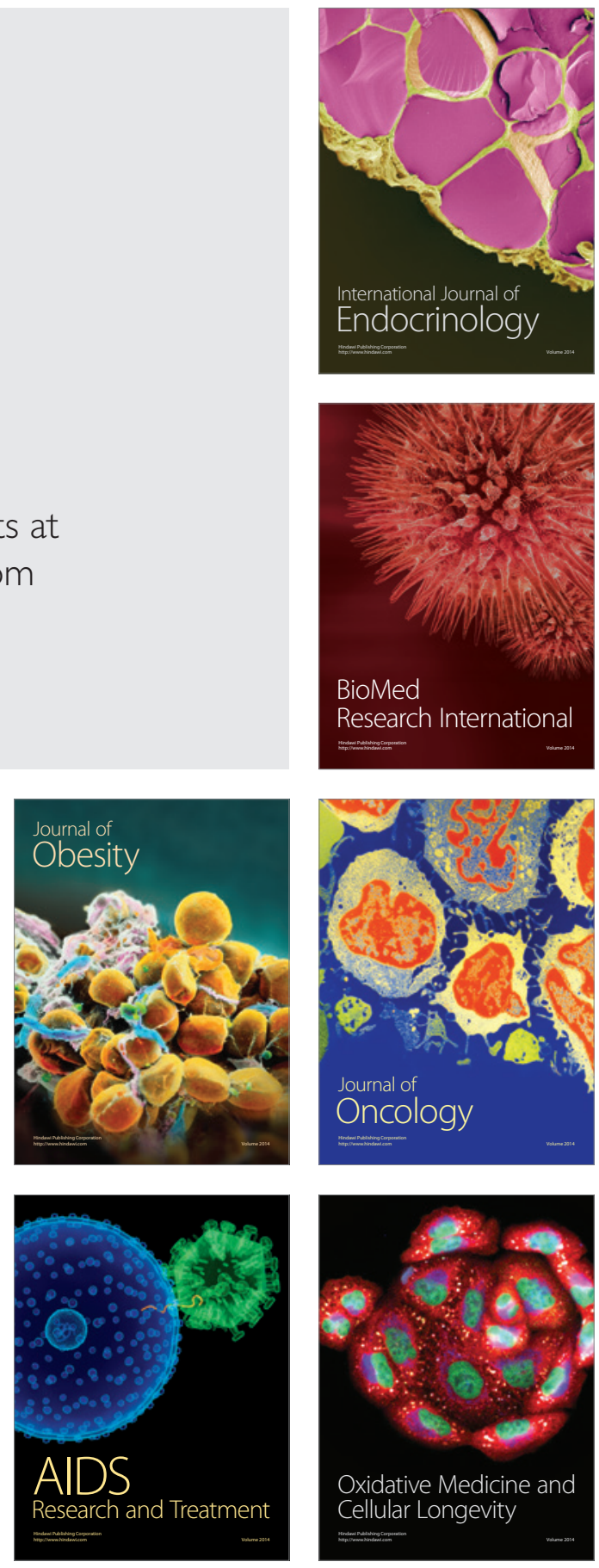